\title{
The Computational Power of Optimization in Online Learning
}

\author{
Elad Hazan \\ Princeton University \\ 35 Olden St, Princeton NJ 08540 \\ ehazan@cs.princeton.edu
}

\author{
Tomer Koren \\ Technion-Israel Institute of Technology \\ Technion City, Haifa 3200003, Israel \\ tomerk@technion.ac.il
}

\begin{abstract}
We consider the fundamental problem of prediction with expert advice where the experts are "optimizable": there is a black-box optimization oracle that can be used to compute, in constant time, the leading expert in retrospect at any point in time. In this setting, we give a novel online algorithm that attains vanishing regret with respect to $N$ experts in total $\widetilde{O}(\sqrt{N})$ computation time. We also give a lower bound showing that this running time cannot be improved (up to log factors) in the oracle model, thereby exhibiting a quadratic speedup as compared to the standard, oracle-free setting where the required time for vanishing regret is $\widetilde{\Theta}(N)$. These results demonstrate an exponential gap between the power of optimization in online learning and its power in statistical learning: in the latter, an optimization oracle - i.e., an efficient empirical risk minimizer-allows to learn a finite hypothesis class of size $N$ in time $O(\log N)$.

We also study the implications of our results to learning in repeated zero-sum games, in a setting where the players have access to oracles that compute, in constant time, their bestresponse to any mixed strategy of their opponent. We show that the runtime required for approximating the minimax value of the game in this setting is $\widetilde{\Theta}(\sqrt{N})$, yielding again a quadratic improvement upon the oracle-free setting, where $\widetilde{\Theta}(N)$ is known to be tight.
\end{abstract}

\section{Categories and Subject Descriptors}

F.2 [Theory of Computation]: Analysis of Algorithms and Problem Complexity; I.2.6 [Artificial Intelligence]: Learning

\section{General Terms}

Algorithms, Theory

\section{Keywords}

Online learning, Optimization oracles, Learning in games, Zero-sum games, Best-response dynamics, Local search

Permission to make digital or hard copies of all or part of this work for personal or classroom use is granted without fee provided that copies are not made or distributed for profit or commercial advantage and that copies bear this notice and the full citation on the first page. Copyrights for components of this work owned by others than ACM must be honored. Abstracting with credit is permitted. To copy otherwise, or republish, to post on servers or to redistribute to lists, requires prior specific permission and/or a fee. Request permissions from Permissions@acm.org.

STOC'16, June 19-21, 2016, Cambridge, MA, USA

(c) 2016 ACM. $978-1-4503-4132-5 / 16 / 06 \ldots \$ 15.00$

http://dx.doi.org/10.1145/2897518.2897536

\section{INTRODUCTION}

Prediction with expert advice is a fundamental model of sequential decision making and online learning in games. This setting is often described as the following repeated game between a player and an adversary: on each round, the player has to pick an expert from a fixed set of $N$ possible experts, the adversary then reveals an arbitrary assignment of losses to the experts, and the player incurs the loss of the expert he chose to follow. The goal of the player is to minimize his $T$-round average regret, defined as the difference between his average loss over $T$ rounds of the game and the average loss of the best expert in that period - the one having the smallest average loss in hindsight. Multiplicative weights algorithms [36, 20; see also 6 for an overview] achieve this goal by maintaining weights over the experts and choosing which expert to follow by sampling proportionally to the weights; the weights are updated from round to round via a multiplicative update rule according to the observed losses.

While multiplicative weights algorithms are very general and provide particularly attractive regret guarantees that scale with $\log N$, they need computation time that grows linearly with $N$ to achieve meaningful average regret. The number of experts $N$ is often exponentially large in applications (think of the number of all possible paths in a graph, or the number of different subsets of a certain ground set), motivating the search for more structured settings where efficient algorithms are possible. Assuming additional structure - such as linearity, convexity, or submodularity of the loss functions - one can typically minimize regret in total poly $(\log N)$ time in many settings of interest [e.g., 44, 34, 7, 28]. However, the basic multiplicative weights algorithm remains the most general and is still widely used.

The improvement in structured settings - most notably in the linear case [34] and in the convex case [44] — often comes from a specialized reduction of the online problem to the offline version of the optimization problem. In other words, efficient online learning is made possible by providing access to an offline optimization oracle over the experts, that allows the player to quickly compute the best performing expert with respect to any given distribution over the adversary's losses. However, in all of these cases, the regret and runtime guarantees of the reduction need the additional structure. Thus, it is natural to ask whether such a drastic improvement in runtime is possible for generic online learning. Specifically, we ask:

What is the runtime required for minimizing regret given a black-box optimization oracle for the experts, without as- 
suming any additional structure? Can one do better than linear time in $N$ ?

In this paper, we give a precise answer to these questions. We show that, surprisingly, an offline optimization oracle gives rise to a substantial, quadratic improvement in the runtime required for convergence of the average regret. We give a new algorithm that is able to minimize regret in total time $\widetilde{O}(\sqrt{N}),{ }^{1}$ and provide a matching lower bound confirming that this is, in general, the best possible. Thus, our results establish a tight characterization of the computational power of black-box optimization in online learning. In particular, unlike in many of the structured settings where poly $(\log N)$ runtime is possible, without imposing additional structure a polynomial dependence on $N$ is inevitable.

Our results demonstrate an exponential gap between the power of optimization in online learning, and its power in statistical learning. It is a simple and well-known fact that for a finite hypothesis class of size $N$ (which corresponds to a set of $N$ experts in the online setting), black-box optimization gives rise to a statistical learning algorithm - often called empirical risk minimization - that needs only $O(\log N)$ examples for learning. Thus, given an offline optimization oracle that optimizes in constant time, statistical learning can be performed in time $O(\log N)$; in contrast, our results show that the complexity of online learning using such an optimization oracle is $\widetilde{\Theta}(\sqrt{N})$. This dramatic gap is surprising due to a long line of work in online learning suggesting that whatever can be done in an offline setting can also be done (efficiently) online.

Finally, we study the implication of our results to repeated game playing in two-player zero-sum games. The analogue of an optimization oracle in this setting is a best-response oracle for each of the players, that allows her to quickly compute the pure action being the best-response to any given mixed strategy of her opponent. In this setting, we consider the problem of approximately solving a zero-sum gamenamely finding a mixed strategy profile with payoff close to the minimax payoff of the game. We show that our new online learning algorithm above, if deployed by each of the players in an $N \times N$ zero-sum game, guarantees convergence to an approximate equilibrium in total $\widetilde{O}(\sqrt{N})$ time. This is, again, a quadratic improvement upon the best possible $\widetilde{\Theta}(N)$ runtime in the oracle-free setting, as established by Grigoriadis and Khachiyan [24] and Freund and Schapire [21]. Interestingly, it turns out that the quadratic improvement is tight for solving zero-sum games as well: we prove that any algorithm would require $\widetilde{\Omega}(\sqrt{N})$ time to approximate the value of a zero-sum game in general, even when given access to powerful best-response oracles.

\subsection{Related Work}

\section{Online-to-offline reductions.}

The most general reduction from regret minimization to optimization was introduced in the influential work of Kalai and Vempala [34] as the Follow-the-Perturbed Leader (FPL) methodology. This technique requires the problem at hand to be embeddable in a low-dimensional space and the cost functions to be linear in that space. ${ }^{2}$ Subsequently, Kakade

\footnotetext{
${ }^{1}$ Here and throughout, we use the $\widetilde{O}(\cdot)$ notation to hide constants and poly-logarithmic factors.

${ }^{2}$ The extension to convex cost functions is straightforward; see, e.g., [27].
}

et al. [33] reduced regret minimization to approximate linear optimization. For general convex functions, the Follow-theRegularized-Leader (FTRL) framework [44, 27] provides a general reduction from online to offline optimization, that often gives dimension-independent convergence rates. Another general reduction was suggested by Kakade and Kalai [32] for the related model of transductive online learning, where future data is partially available to the player (in the form of unlabeled examples).

Without a fully generic reduction from online learning to optimization, specialized online variants for numerous optimization scenarios have been explored. This includes efficient regret-minimization algorithms for online variance minimization [43], routing in networks [7], online permutations and ranking [31], online planning [18], matrix completion [30], online submodular minimization [28], contextual bandits $[17,4]$, and many more.

\section{Computational tradeoffs in learning.}

Tradeoffs between sample complexity and computation in statistical learning have been studied intensively in recent years [e.g., 3, 41, 42]. However, the adversarial setting of online learning, which is our main focus in this paper, did not receive a similar attention. One notable exception is the seminal paper of Blum [8] who showed that, under certain cryptographic assumptions, there exists an hypothesis class which is computationally hard to learn in the online mistake bound model but is non-properly learnable in polynomial time in the PAC model. ${ }^{3}$ In our terminology, Blum's result show that online learning might require $\omega($ poly $(\log N))$ time, even in a case where offline optimization can be performed in poly $(\log N)$ time, albeit non-properly (i.e., the optimization oracle is allowed to return a prediction rule which is not necessarily one of the $N$ experts).

\section{Solution of zero-sum games.}

The computation of equilibria in zero-sum games is known to be equivalent to linear programming, as was first observed by von-Neumann [2]. A basic and well-studied question in game theory is the study of rational strategies that converge to equilibria (see [39] for an overview). Freund and Schapire [21] showed that in zero-sum games, no-regret algorithms converge to equilibrium. Hart and Mas-Colell [26] studied convergence of no-regret algorithms to correlated equilibria in more general games; Even-dar et al. [19] analyzed convergence to equilibria in concave games. Grigoriadis and Khachiyan [24] were the first to observe that zero-sum games can be solved in total time sublinear in the size of the game matrix.

Game dynamics that rely on best-response computations have been a topic of extensive research for more than half a century, since the early days of game theory. Within this line of work, perhaps the most prominent dynamic is the "fictitious play" algorithm, in which both players repeatedly follow their best-response to the empirical distribution of their opponent's past plays. This simple and natural dynamic was first proposed by Brown [11], shown to converge to equilibrium in two-player zero-sum games by Robinson [40], and was extensively studied ever since (see e.g., [10, 15]

\footnotetext{
3 "Non-proper learning" means that the algorithm is allowed to return an hypothesis outside of the hypothesis class it competes with.
} 
and the references therein). Another related dynamic, put forth by Hannan [25] and popularized by Kalai and Vempala [34], is based on perturbed (i.e., noisy) best-responses.

We remark that since the early works of Grigoriadis and Khachiyan [24] and Freund and Schapire [21], faster algorithms for approximating equilibria in zero-sum games have been proposed [e.g., 38, 16]. However, the improvements there are in terms of the approximation parameter $\epsilon$ rather than the size of the game $N$. It is a simple folklore fact that using only value oracle access to the game matrix, any algorithm for approximating the equilibrium must run in time $\Omega(N)$; see, e.g., [13].

\section{FORMAL SETUP AND STATEMENT OF RESULTS}

We now formalize our computational oracle-based model for learning in games - a setting which we call "Optimizable Experts". The model is essentially the classic online learning model of prediction with expert advice augmented with an offline optimization oracle.

Prediction with expert advice can be described as a repeated game between a player and an adversary, characterized by a finite set $\mathcal{X}$ of $N$ experts for the player to choose from, a set $\mathcal{Y}$ of actions for the adversary, and a loss function $\ell: \mathcal{X} \times \mathcal{Y} \mapsto[0,1]$. First, before the game begins, the adversary picks an arbitrary sequence $y_{1}, y_{2}, \ldots$ of actions from $\mathcal{Y} .{ }^{4}$ On each round $t=1,2, \ldots$, of the game, the player has to choose (possibly at random) an expert $x_{t} \in \mathcal{X}$, the adversary then reveals his action $y_{t} \in \mathcal{Y}$ and the player incurs the loss $\ell\left(x_{t}, y_{t}\right)$. The goal of the player is to minimize his expected average regret over $T$ rounds, defined as

$$
R(T)=\mathbb{E}\left[\frac{1}{T} \sum_{t=1}^{T} \ell\left(x_{t}, y_{t}\right)\right]-\min _{x \in \mathcal{X}} \frac{1}{T} \sum_{t=1}^{T} \ell\left(x, y_{t}\right)
$$

Here, the expectation is taken with respect to the randomness in the choices of the player.

In the optimizable experts model, we assume that the loss function $\ell$ is initially unknown to the player, and allow her to access $\ell$ by means of two oracles: Val and Opt. The first oracle simply computes for each pair of actions $(x, y)$ the respective loss $\ell(x, y)$ incurred by expert $x$ when the adversary plays the action $y$.

DeFinition (value oracle). A value oracle is a procedure Val : $\mathcal{X} \times \mathcal{Y} \mapsto[0,1]$ that for any action pair $x \in \mathcal{X}, y \in \mathcal{Y}$, returns the loss value $\ell(x, y)$ in time $O(1)$; that is,

$$
\forall x \in \mathcal{X}, y \in \mathcal{Y}, \quad \operatorname{Val}(x, y)=\ell(x, y) .
$$

The second oracle is far more powerful, and allows the player to quickly compute the best performing expert with respect to any given distribution over actions from $\mathcal{Y}$ (i.e., any mixed strategy of the adversary).

DEFINITION (optimization oracle). An optimization oracle is a procedure Opt that receives as input a distribution $q \in \Delta(\mathcal{Y})$, represented as a list of atoms $\left\{\left(i, q_{i}\right): q_{i}>0\right\}$,

\footnotetext{
${ }^{4}$ Such an adversary is called oblivious, since it cannot react to the decisions of the player as the game progresses. We henceforth assume an oblivious adversary, and relax this assumption later in Section 4.
}

and returns a best performing expert with respect to $q$ (with ties broken arbitrarily), namely

$$
\forall q \in \Delta(\mathcal{Y}), \quad \operatorname{Opt}(q) \in \underset{x \in \mathcal{X}}{\arg \min } \mathbb{E}_{y \sim q}[\ell(x, y)] .
$$

The oracle Opt runs in time $O(1)$ on any input.

Recall that our goal in this paper is to evaluate online algorithms by their runtime complexity. To this end, it is natural to consider the running time it takes for the average regret of the player to drop below some specified target threshold. ${ }^{5}$ Namely, for a given $\epsilon>0$, we will be interested in the total computational cost (as opposed to the number of rounds) required for the player to ensure that $R(T)<\epsilon$, as a function of $N$ and $\epsilon$. Notice that the number of rounds $T$ required to meet the latter goal is implicit in this view, and only indirectly affects the total runtime.

\subsection{Main Results}

We can now state the main results of the paper: a tight characterization of the runtime required for the player to converge to $\epsilon$ expected average regret in the optimizable experts model.

THEOREM 1. In the optimizable experts model, there exists an algorithm that for any $\epsilon>0$, guarantees an expected average regret of at most $\epsilon$ with total runtime of $\widetilde{O}\left(\sqrt{N} / \epsilon^{2}\right)$. Specifically, Algorithm 2 (see Section 3.2) achieves $\widetilde{O}\left(N^{1 / 4} / \sqrt{T}\right)$ expected average regret over $T$ rounds, and runs in $\widetilde{O}(1)$ time per round.

The dependence on the number of experts $N$ in the above result is tight, as the following theorem shows.

THEOREM 2. Any (randomized) algorithm in the optimizable experts model cannot guarantee an expected average regret smaller than $\frac{1}{2}$ in total time better than $O(\sqrt{N})$.

In other words, we exhibit a quadratic improvement in the total runtime required for the average regret to converge, as compared to standard multiplicative weights schemes that require $\widetilde{O}\left(N / \epsilon^{2}\right)$ time, and this improvement is the best possible. Granted, the regret bound attained by the algorithm is inferior to those achieved by multiplicative weights methods, that depend on $N$ only logarithmically; however, when we consider the total computational cost required for convergence, the substantial improvement is evident.

Our upper bound actually applies to a model more general than the optimizable experts model, where instead of having access to an optimization oracle, the player receives information about the leading expert on each round of the game. Namely, in this model the player observes at the end of round $t$ the leader

$$
x_{t}^{\star}=\underset{x \in \mathcal{X}}{\arg \min } \sum_{s=1}^{t} \ell\left(x, y_{s}\right)
$$

as part of the feedback. This is indeed a more general model, as the leader $x_{t}^{\star}$ can be computed in the oracle model in amortized $O(1)$ time, simply by calling $\operatorname{Opt}\left(y_{1}, \ldots, y_{t}\right)$. (The list of actions $y_{1}, \ldots, y_{t}$ played by the adversary can be maintained in an online fashion in $O(1)$ time per round.) Our lower bound, however, applies even when the player has access to an optimization oracle in its full power.

\footnotetext{
${ }^{5}$ This is indeed the appropriate criterion in algorithmic applications of online learning methods.
} 
Finally, we mention a simple corollary of Theorem 2: we obtain that the time required to attain vanishing average regret in online Lipschitz-continuous optimization in Euclidean space is exponential in the dimension, even when an oracle for the corresponding offline optimization problem is at hand. For the precise statement of this result, see [29].

\subsection{Zero-sum Games with Best-response Oracles}

In this section we present the implications of our results for repeated game playing in two-player zero-sum games. Before we can state the results, we first recall the basic notions of zero-sum games and describe the setting formally.

A two-player zero-sum game is specified by a matrix $G \in[0,1]^{N \times N}$, in which the rows correspond to the (pure) strategies of the first player, called the row player, while the columns correspond to strategies of the second player, called the column player. For simplicity, we restrict the attention to games in which both players have $N$ pure strategies to choose from; our results below can be readily extended to deal with games of general (finite) size. A mixed strategy of the row player is a distribution $p \in \Delta_{N}$ over the rows of $G$; similarly, a mixed strategy for the column player is a distribution $q \in \Delta_{N}$ over the columns. For players playing strategies $(p, q)$, the loss (respectively payoff) suffered by the row (respectively column) player is given by $p^{\top} G q$. A pair of mixed strategies $(p, q)$ is said to be an approximate equilibrium, if for both players there is almost no incentive in deviating from the strategies $p$ and $q$. Formally, $(p, q)$ is an $\epsilon$-equilibrium if and only if

$$
\forall 1 \leqslant i, j \leqslant N, \quad p^{\top} G e_{j}-\epsilon \leqslant p^{\top} G q \leqslant e_{i}^{\top} G q+\epsilon .
$$

Here and throughout, $e_{i}$ stands for the $i$ 'th standard basis vector, namely a vector with 1 in its $i$ 'th coordinate and zeros elsewhere. The celebrated von-Neumann minimax theorem asserts that for any zero-sum game there exists an exact equilibrium (i.e., with $\epsilon=0$ ) and it has a unique value, given by

$$
\lambda(G)=\min _{p \in \Delta_{N}} \max _{q \in \Delta_{N}} p^{\top} G q .
$$

A repeated zero-sum game is an iterative process in which the two players simultaneously announce their strategies, and suffer loss (or receive payoff) accordingly. Given $\epsilon>0$, the goal of the players in the repeated game is to converge, as quickly as possible, to an $\epsilon$-equilibrium; in this paper, we will be interested in the total runtime required for the players to reach an $\epsilon$-equilibrium, rather than the total number of game rounds required to do so.

We assume that the players do not know the game matrix $G$ in advance, and may only access it through two types of oracles, which are very similar to the ones we defined in the online learning model. The first and most natural oracle allows the player to query the payoff for any pair of pure strategies (i.e., a pure strategy profile) in constant time. Formally,

Definition (value oracle). A value oracle for a zero-sum game described by a matrix $G \in[0,1]^{N \times N}$ is a procedure Val that accepts row and column indices $i, j$ as input and returns the game value for the pure strategy profile $(i, j)$, namely:

$$
\forall 1 \leqslant i, j \leqslant N, \quad \operatorname{Val}(i, j)=G_{i, j} .
$$

The value oracle runs in time $O(1)$ on any valid input.

The other oracle we consider is the analogue of an optimization oracle in the context of games. For each of the players, a best-response oracle is a procedure that computes the player's best response (pure) strategy to any mixed strategy of his opponent, given as input.

Definition (best-response oracle). A best-response oracle for the row player in a zero-sum game described by a matrix $G \in[0,1]^{N \times N}$, is a procedure $\mathrm{BR}^{1}$ that receives as input a distribution $q \in \Delta_{N}$, represented as a list of atoms $\left\{\left(i, q_{i}\right): q_{i}>0\right\}$, and computes

$$
\forall q \in \Delta_{N}, \quad \operatorname{BR}^{1}(q) \in \underset{1 \leqslant i \leqslant N}{\arg \min } \mathrm{e}_{i}^{\top} G q
$$

with ties broken arbitrarily. Similarly, a best-response oracle $\mathrm{BR}^{2}$ for the column player accepts as input a $p \in \Delta_{N}$ represented as a list $\left\{\left(i, p_{i}\right): p_{i}>0\right\}$, and computes

$$
\forall p \in \Delta_{N}, \quad \operatorname{BR}^{2}(p) \in \underset{1 \leqslant j \leqslant N}{\arg \max } p^{\top} G \mathrm{e}_{j} .
$$

Both best-response oracles return in time $O(1)$ on any input.

Our main results regarding the runtime required to converge to an approximate equilibrium in zero-sum games with best-response oracles, are the following.

ThEOREM 3. There exists an algorithm (see Algorithm 6 in Section 4) that for any zero-sum game with [0,1] payoffs and for any $\epsilon>0$, terminates in time $\widetilde{O}\left(\sqrt{N} / \epsilon^{2}\right)$ and outputs with high probability an $\epsilon$-approximate equilibrium.

THEOREM 4. Any (randomized) algorithm for approximating the equilibrium of $N \times N$ zero-sum games with bestresponse oracles cannot guarantee with probability greater than $\frac{2}{3}$ that the average payoff of the row player is at most $\frac{1}{4}$-away from its value at equilibrium in total time better than $\widetilde{O}(\sqrt{N})$.

As indicated earlier, these results show that best-response oracles in repeated game playing give rise again to a quadratic improvement in the runtime required for solving zero-sum games, as compared to the best possible runtime to do so without an access to best-response oracles, which scales linearly with $N[24,21]$.

The algorithm deployed in Theorem 3 above is a very natural one: it simulates a repeated game where both players play a slight modification of the regret minimization algorithm of Theorem 1, and the best-response oracle of each player serves as the optimization oracle required for the online algorithm.

Due to space constraints, we omit the proofs Theorems 3 and 4 from this extended abstract and defer details to [29].

\subsection{Overview of the Approach and Techniques}

We now outline the main ideas leading to the quadratic improvement in runtime achieved by our online algorithm of Theorem 1. Intuitively, the challenge is to reduce the number of "effective" experts quadratically, from $N$ to roughly $\sqrt{N}$. Since we have an optimization oracle at our disposal, it is natural to focus on the set of "leaders"- those experts that have been best at some point in history - and try to reduce the complexity of the online problem to scale with the number of such leaders. This set is natural considering our computational concerns: the algorithm can obtain 
information on the leaders at almost no cost (using the optimization oracle, it can compute the leader on each round in only $O(1)$ time per round), resulting with a potentially substantial advantage in terms of runtime.

First, suppose that there is a small number of leaders throughout the game, say $L=O(\sqrt{N})$. Then, intuitively, the problem we face is easy: if we knew the identity of those leaders in advance, our regret would scale with $L$ and be independent of the total number of experts $N$. As a result, using standard multiplicative weights techniques we would be able to attain vanishing regret in total time that depends linearly on $L$, and in case $L=O(\sqrt{N})$ we would be done. When the leaders are not known in advance, one could appeal to various techniques that were designed to deal with experts problems in which the set of experts evolves over time [e.g., 22, 9, 35, 23]. However, the per-round runtime of all of these methods is linear in $L$, which is prohibitive for our purposes. We remark that the simple "follow the leader" algorithm, that simply chooses the most recent leader on each round of the game, is not guaranteed to perform well in this case: the regret of this algorithm scales with the number of times the leader switches - rather than the number of distinct leaders - that might grow linearly with $T$ even when there are few active leaders.

A main component in our approach is a novel online learning algorithm, called LEADERS, that keeps track of the leaders in an online game, and attains $\widetilde{O}(\sqrt{L / T})$ average regret in expectation with $\widetilde{O}(1)$ runtime per round. The algorithm, that we describe in detail in Section 3.1, queries the oracles only $\widetilde{O}(1)$ times per iteration and thus can be implemented efficiently. More formally,

ThEOREM 5. The expected $T$-round average regret of the LEADERS algorithm is at most $O(\sqrt{(L / T) \log (L T)})$, where $L$ is an upper bound over the total number of distinct leaders during throughout the game. The algorithm can be implemented in $\widetilde{O}(1)$ time per round in the optimizable experts model.

As far as we know, this technique is new to the theory of regret minimization and may be of independent interest. In a sense, it is a partial-information algorithm: it is allowed to use only a small fraction of the feedback signal (i.e., read a small fraction of the loss values) on each round, due to the time restrictions. Nevertheless, its regret guarantee can be shown to be optimal in terms of the number of leaders $L$, even when removing the computational constraints! The new algorithm is based on running in parallel a hierarchy of multiplicative-updates algorithms with varying look-back windows for keeping track of recent leaders.

But what happens if there are many leaders, say $L=$ $\Omega(\sqrt{N})$ ? In this case, we can incorporate random guessing: if we sample about $\sqrt{N}$ experts, with nice probability one of them would be among the "top" $\sqrt{N}$ leaders. By competing with this small random set of experts, we can keep the regret under control, up to the point in time where at most $\sqrt{N}$ leaders remain active (in the sense that they appear as leaders at some later time). In essence, this observation allows us to reduce the effective number of leaders back to the order of $\sqrt{N}$ and use the approach detailed above even when $L=\Omega(\sqrt{N})$, putting the LEADERS algorithm into action at the point in time where the top $\sqrt{N}$ leader is encountered (without actually knowing when exactly this event occurs).
In order to apply our algorithm to repeated two-player zero-sum games and obtain Theorem 3, we first show how it can be adapted to minimize regret even when used against an adaptive adversary, that can react to the decisions of the algorithm (as is the case in repeated games). Then, via standard techniques [21], we show that the quadratic speedup we achieved in the online learning setting translates to similar speedup in the solution of zero-sum games. In a nutshell, we let both players use our online regret-minimization algorithm for picking their strategies on each round of the game, where they use their best-response oracles to fill the role of the optimization oracle in the optimizable experts model.

Our lower bounds (i.e., Theorems 2 and 4) are based on information-theoretic arguments, which can be turned into running time lower bounds in our oracle-based computational model. In particular, the lower bound for zero-sum games is based on a reduction to a problem investigated by Aldous [5] and revisited years later by Aaronson [1], and reveals interesting connections between the solution of zerosum games and local-search problems. Aldous investigated the hardness of local-search problems and gave an explicit example of an efficiently-representable (random) function which is hard to minimize over its domain, even with access to a local improvement oracle. (A local improvement oracle improves upon a given solution by searching in its local neighborhood.) Our reduction constructs a zero-sum game in which a best-response query amounts to a localimprovement step, and translates Aldous' query-complexity lower bound to a runtime lower bound in our model.

Interestingly, the connection to local-search problems is also visible in our algorithmic results: our algorithm for learning with optimizable experts (Algorithm 2) involves guessing a "top $\sqrt{N}$ " solution (i.e., a leader) and making $\sqrt{N}$ local-improvement steps to this solution (i.e., tracking the finalist leaders all the way to the final leader). This is reminiscent of a classical randomized algorithm for localsearch, pointed out by Aldous [5].

\section{ALGORITHMS FOR OPTIMIZABLE EXPERTS}

In this section we develop our algorithms for online learning in the optimizable experts model. Recall that we assume a more general setting where there is no optimization oracle, but instead the player observes after each round $t$ the identity of the leader $x_{t}^{\star}$ (see Eq. (1)) as part of the feedback on that round. Thus, in what follows we assume that the leader $x_{t}^{\star}$ is known immediately after round $t$ with no additional computational costs, and do not require the oracle Opt any further.

To simplify the presentation, we introduce the following notation. We fix an horizon $T>0$ and denote by $f_{1}, \ldots, f_{T}$ the sequence of loss functions induced by the actions $y_{1}, \ldots, y_{T}$ chosen by the adversary, where $f_{t}(\cdot)=$ $\ell\left(\cdot, y_{t}\right)$ for all $t$; notice that the resulting sequence $f_{1}, \ldots, f_{T}$ is a completely arbitrary sequence of loss functions over $\mathcal{X}$, as both $\ell$ and the $y_{t}$ 's are chosen adversarially. We also fix the set of experts to $\mathcal{X}=[N]=\{1, \ldots, N\}$, identifying each expert with its serial index.

\subsection{The Leaders Algorithm}

We begin by describing the main technique in our algorithmic results - the LEADERS algorithm - which is key to 
PARAMETERS: $L, T$

1. Set $\eta_{0}=\sqrt{\log (2 L T)}$ and $\nu=2 \eta_{0} \sqrt{L / T}$

2. For all $r=1, \ldots,\left\lceil\log _{2} L\right\rceil$ and $s=1, \ldots,\left\lceil\log _{2} T\right\rceil$, initialize an instance $\mathcal{A}_{r, s}$ of $\mathrm{MW}^{3}(k, \eta, \gamma)$ with $k=2^{r}$, $\eta=\eta_{0} / \sqrt{2^{r+s}}$ and $\gamma=\frac{1}{T}$

3. Initialize an instance $\mathcal{A}$ of $\mathrm{MW}^{1}\left(\nu, \frac{1}{T}\right)$ algorithm on the $\mathcal{A}_{r, s}$ 's as experts

4. For $t=1,2, \ldots$ :

(a) Play the prediction $x_{t}$ of $\mathcal{A}$

(b) Observe feedback $f_{t}$ and the leader $x_{t}^{\star}$, and use it to update all algorithms $\mathcal{A}_{r, s}$

\section{Algorithm 1: The Leaders algorithm.}

proving Theorem 1. LEADERS is an online algorithm designed to perform well in online learning problems with a small number of leaders, both in terms of average regret and computational costs. The algorithm makes use of the information on the leaders $x_{1}^{\star}, x_{2}^{\star}, \ldots$ received as feedback to save computation time, and can be made to run in almost constant time per round (up to logarithmic factors).

The LEADERS algorithm is presented in Algorithm 1. In the following theorem we state its guarantees; the theorem gives a slightly more general statement than the one presented earlier in Theorem 5, that we require for the proof of our main result.

THEOREM 6. Assume that LEADERS is used for prediction with expert advice (with leaders feedback) against loss functions $f_{1}, f_{2}, \ldots:[N] \mapsto[0,1]$, and that the total number of distinct leaders during a certain time period $t_{0}<t \leqslant t_{1}$ whose length is bounded by $T$, is at most L. Then, provided the numbers $L$ and $T$ are given as input, the algorithm obtains the following regret guarantee:

$$
\begin{aligned}
& \mathbb{E}\left[\sum_{t=t_{0}+1}^{t_{1}} f_{t}\left(x_{t}\right)\right]-\left(\sum_{t=1}^{t_{1}} f_{t}\left(x_{t_{1}}^{\star}\right)-\sum_{t=1}^{t_{0}} f_{t}\left(x_{t_{0}}^{\star}\right)\right) \\
& \leqslant 25 \sqrt{L T \log (2 L T)} .
\end{aligned}
$$

The algorithm can be implemented to run in time $O\left(\log ^{2}(L) \log (T)\right)$ per round.

Algorithm 2 relies on two simpler online algorithms - the $\mathrm{MW}^{1}$ and $\mathrm{MW}^{3}$ algorithms - that we describe in detail later on in this section (see Section 3.3, where we also discuss an algorithm called $\mathrm{MW}^{2}$ ). These two algorithms are variants of the standard multiplicative weights (MW) method for prediction with expert advice. $\mathrm{MW}^{1}$ is a rather simple adaptation of MW which is able to guarantee bounded regret in any time interval of predefined length:

Lemma 11. Suppose that $\mathrm{MW}^{1}$ (Algorithm 3 below) is used for prediction with expert advice, against an arbitrary sequence of loss functions $f_{1}, f_{2}, \ldots:[N] \mapsto[0,1]$ over $N$ experts. Then, for $\gamma=\frac{1}{T}$ and any $\eta>0$, its sequence of predictions $x_{1}, x_{2}, \ldots$ satisfies

$$
\mathbb{E}\left[\sum_{t=t_{0}}^{t_{1}} f_{t}\left(x_{t}\right)\right]-\min _{x \in[N]} \sum_{t=t_{0}}^{t_{1}} f_{t}(x) \leqslant \frac{2 \log (N T)}{\eta}+\eta T
$$

in any time interval $\left\{t_{0}, \ldots, t_{1}\right\}$ of length at most $T$. The algorithm can be implemented to run in $O(N)$ time per round.
The $\mathrm{MW}^{3}$ algorithm a "sliding window" version of $\mathrm{MW}^{1}$, that given a parameter $k>0$, maintains a buffer of $k$ experts that were recently "activated"; in our context, an expert is activated on round $t$ if it is the leader at the end of that round. $\mathrm{MW}^{3}$ competes (in terms of regret) with the $k$ most recent activated experts as long as they remain in the buffer. Formally,

Lemma 13. Suppose that $\mathrm{MW}^{3}$ (Algorithm 5 below) is used for prediction with expert advice, against an arbitrary sequence of loss functions $f_{1}, f_{2}, \ldots:[N] \mapsto[0,1]$ over $N$ experts. Assume that expert $x^{\star} \in[N]$ was activated on round $t_{0}$, and from that point until round $t_{1}$ there were no more than $k$ different activated experts (including $x^{\star}$ itself). Then, for $\gamma=\frac{1}{T}$ and any $\eta>0$, the predictions $x_{1}, x_{2}, \ldots$ of the algorithm satisfy

$$
\mathbb{E}\left[\sum_{t=t_{0}^{\prime}+1}^{t_{1}^{\prime}} f_{t}\left(x_{t}\right)\right]-\sum_{t=t_{0}^{\prime}+1}^{t_{1}^{\prime}} f_{t}\left(x^{\star}\right) \leqslant \frac{4 \log (k T)}{\eta}+\eta k T
$$

in any time interval $\left[t_{0}^{\prime}, t_{1}^{\prime}\right] \subseteq\left[t_{0}, t_{1}\right]$ of length at most $T$. Furthermore, the algorithm can be implemented to run in time $\widetilde{O}(1)$ per round.

For the analysis of Algorithm 1, we require a few definitions. We let $I=\left\{t_{0}+1, \ldots, t_{1}\right\}$ denote the time interval under consideration. For all $t \in I$, we denote by $S_{t}=\left\{x_{t_{0}+1}^{\star}, \ldots, x_{t}^{\star}\right\}$ the set of all leaders encountered since round $t_{0}+1$ up to and including round $t$; for completeness we also define $S_{t_{0}}=\varnothing$. The theorem's assumption then implies that $\left|S_{t_{0}}\right| \leqslant \ldots \leqslant\left|S_{t_{1}}\right| \leqslant L$. For a set of experts $S \subseteq[N]$, we let $\tau(S)=\max \left\{t \in I: x_{t}^{\star} \in S\right\}$ be the last round in which one of the experts in $S$ occurs as a leader. In other words, after round $\tau(S)$, the leaders in $S$ have "died out" and no longer appear as leaders.

Next, we split $I$ into epochs $I_{1}, I_{2}, \ldots$, where the $i$ 'th epoch $I_{i}=\left\{\tau_{i}+1, \ldots, \tau_{i+1}\right\}$ spans between rounds $\tau_{i}+1$ and $\tau_{i+1}$, and $\tau_{i}$ is defined recursively by $\tau_{1}=t_{0}$ and $\tau_{i+1}=\tau\left(S_{\tau_{i}+1}\right)$ for all $i=1,2, \ldots$ In words, $S_{\tau_{i}+1}$ is the set of leaders encountered by the beginning of epoch $i$, and this epoch ends once all leaders in this set have died out. Let $m$ denote the number of resulting epochs (notice that $m \leqslant L$, as at least one leader dies out in each of the epochs). For each $i=1, \ldots, m$, let $T_{i}$ denote the length of the $i$ 'th epoch, namely $m=\max \left\{i: \tau_{i}<t_{1}\right\}$, and let $z_{i}^{\star}=x_{\tau_{i}}^{\star}$ be the leader at the end of epoch $i$. Finally, for each epoch $i=1, \ldots, m$ we let $C_{i}=S_{\tau_{i}+1} \backslash S_{\tau_{i-1}+1}$ denote the set of leaders that have died out during the epoch, and for technical convenience we also define $C_{0}=C_{m+1}=\varnothing$; notice that $C_{1}, \ldots, C_{m}$ is a partition of the set $S_{t_{1}}$ of all leaders, so in particular $\left|C_{1}\right|+\cdots+\left|C_{m}\right| \leqslant L$. See Fig. 1 for an illustration of the definitions.

Our first lemma states that minimizing regret in each epoch $i$ with respect to the leader $z_{i}^{\star}$ at the end of the epoch, also guarantees low regret with respect to the overall leader $x_{t_{1}}^{\star}$. It is a variant of the "Follow The Leader, Be The Leader" lemma [34].

LEMMA 7. Following the epoch's leader yields no regret, in the sense that

$$
\sum_{i=1}^{m} \sum_{t \in I_{i}} f_{t}\left(z_{i}^{\star}\right) \leqslant \sum_{t=1}^{t_{1}} f_{t}\left(x_{t_{1}}^{\star}\right)-\sum_{t=1}^{t_{0}} f_{t}\left(x_{t_{0}}^{\star}\right) .
$$




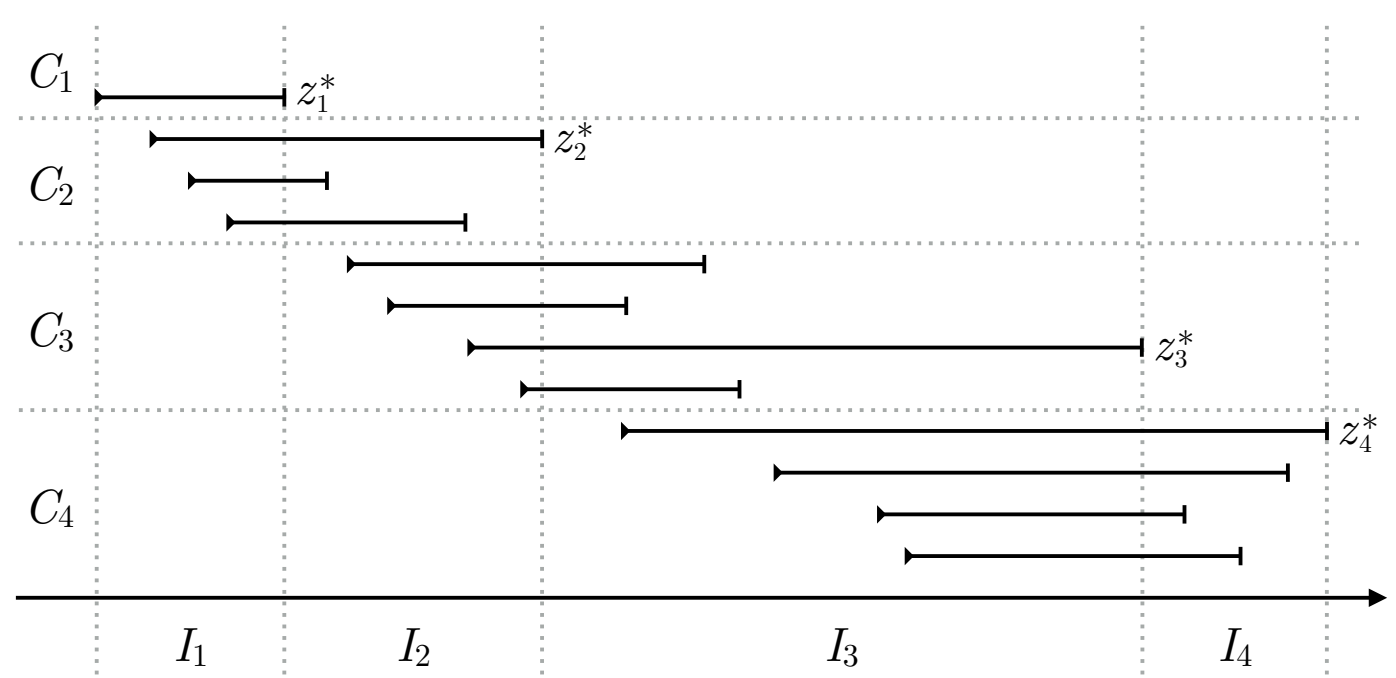

Figure 1: An illustration of the key definitions in the analysis of Algorithm 1. Each expert is represented by a horizontal segment, which signifies the time interval between the expert's first and last appearances as leader (the experts are sorted by their first time of appearance as leaders). The resulting partition $C_{1}, \ldots, C_{4}$ of the experts and the induced epochs $I_{1}, \ldots, I_{4}$ are indicated by the dotted lines.

Proof. Let $I_{0}=\left\{1, \ldots, t_{0}\right\}$ and $z_{0}^{\star}=x_{t_{0}}^{\star}$. We will prove by induction on $m \geqslant 0$ that

$$
\sum_{i=0}^{m} \sum_{t \in I_{i}} f_{t}\left(z_{i}^{\star}\right) \leqslant \sum_{i=0}^{m} \sum_{t \in I_{i}} f_{t}\left(z_{m}^{\star}\right) .
$$

This inequality would imply the lemma, as $z_{m}^{\star}=x_{t_{1}}^{\star}$. For $m=0$, our claim is trivial as both sides of Eq. (2) are equal. Now, assuming that Eq. (2) holds for $m-1$, we have

$$
\begin{aligned}
\sum_{i=0}^{m-1} \sum_{t \in I_{i}} f_{t}\left(z_{i}^{\star}\right) & \leqslant \sum_{i=0}^{m-1} \sum_{t \in I_{i}} f_{t}\left(z_{m-1}^{\star}\right) \quad \text { (induction) } \\
& \leqslant \sum_{i=0}^{m-1} \sum_{t \in I_{i}} f_{t}\left(z_{m}^{\star}\right)
\end{aligned}
$$

since by definition $z_{m-1}^{\star}$ performs better than any other expert, and in particular than $z_{m}^{\star}$, throughout the first $m-1$ epochs. Adding the term $\sum_{t \in I_{m}} f_{t}\left(z_{m}^{\star}\right)$ to both sides of the above inequality, we obtain Eq. (2).

Next, we identify a key property of our partition to epochs.

LEMma 8. For all epochs $i$, it holds that $z_{i}^{\star} \in C_{i}$. In addition, any leader encountered during the lifetime of $z_{i}^{\star}$ as leader (i.e., between its first and last appearances in the sequence $x_{t_{0}+1}^{\star}, \ldots, x_{t_{1}}^{\star}$ of leaders) must be a member of $C_{i-1} \cup C_{i} \cup C_{i+1}$

Proof. Consider epoch $i$ and the leader $z_{i}^{\star}$ at the end of this epoch. To see that $z_{i}^{\star} \in C_{i}$, recall that the $i$ 'th epoch ends right after the leaders in $C_{i}$ have all died out, so the leader at the end of this epoch must be a member of the latter set. This also means that $z_{i}^{\star}$ was first encountered not before epoch $i-1$ (in fact, even not on the first round of that epoch), and the last time it was a leader was on the last round of epoch $i$ (see Fig. 1). In particular, throughout the lifetime of $z_{i}^{\star}$ as leader, only the experts in $C_{i-1} \cup C_{i} \cup C_{i+1}$ could have appeared as leaders.
We are now ready to analyze the regret in a certain epoch $i$ with respect to its leader $z_{i}^{\star}$. To this end, we define $k_{i}=$ $\left|C_{i-1}\right|+\left|C_{i}\right|+\left|C_{i+1}\right|$ and consider the $\mathrm{MW}^{3}$ instance $\mathcal{A}^{(i)}=$ $\mathcal{A}_{r_{i}, s_{i}}$, where $r_{i}=\left\lceil\log _{2} k_{i}\right\rceil$ and $s_{i}=\left\lceil\log _{2} T_{i}\right\rceil$ (note that $1 \leqslant r_{i} \leqslant\left\lceil\log _{2} L\right\rceil$ and $\left.1 \leqslant s_{i} \leqslant\left\lceil\log _{2} T\right\rceil\right)$. The following lemma shows that the regret of the algorithm $\mathcal{A}^{(i)}$ in epoch $i$ can be bounded in terms of the quantity $k_{i}$. Below, we use $z_{t}^{(i)}$ to denote the decision of $\mathcal{A}^{(i)}$ on round $t$.

LEMMA 9. The cumulative expected regret of the algorithm $\mathcal{A}^{(i)}$ throughout epoch $i$, with respect to the leader $z_{i}^{\star}$ at the end of this epoch, has

$$
\mathbb{E}\left[\sum_{t \in I_{i}} f_{t}\left(z_{t}^{(i)}\right)\right]-\sum_{t \in I_{i}} f_{t}\left(z_{i}^{\star}\right) \leqslant 10 \sqrt{k_{i} T_{i} \log (2 L T)} .
$$

Proof. Recall that $\mathcal{A}^{(i)}$ has a buffer of size $q_{i}=2^{r_{i}}$ and step size $\eta_{i}=\sqrt{\log (2 L T) / 2^{r_{i}+s_{i}}}$. Now, from Lemma 8 we know that $z_{i}^{\star} \in C_{i}$, which means that $z_{i}^{\star}$ first appeared as leader either on or before the first round of epoch $i$. Also, the same lemma states that the number of distinct leaders that were encountered throughout the lifetime of $z_{i}^{\star}$ (including $z_{i}^{\star}$ itself) is at most $\left|C_{i-1} \cup C_{i} \cup C_{i+1}\right|=k_{i} \leqslant q_{i}$, namely no more than the size of $\mathcal{A}^{(i)}$ 's buffer. Hence, applying Lemma 13 to epoch $i$, we have

$$
\mathbb{E}\left[\sum_{t \in I_{i}} f_{t}\left(z_{t}^{(i)}\right)\right]-\sum_{t \in I_{i}} f_{t}\left(z_{i}^{\star}\right) \leqslant \frac{4 \log (2 L T)}{\eta_{i}}+\eta_{i} q_{i} T_{i},
$$

where we have used $q_{i} \leqslant 2 L$ and $T_{i} \leqslant T$ to bound the logarithmic term. Now, note that $q_{i} \leqslant 2 k_{i}$ and $\sqrt{\log (2 L T) / 4 k_{i} T_{i}} \leqslant \eta_{i} \leqslant \sqrt{\log (2 L T) / k_{i} T_{i}}$, which follow from $k_{i} \leqslant 2^{r_{i}} \leqslant 2 k_{i}$ and $T_{i} \leqslant 2^{s_{i}} \leqslant 2 T_{i}$. Plugging into the above bound, we obtain the lemma.

Our final lemma analyzes the MW algorithm $\mathcal{A}$, and shows that it obtains low regret against the algorithm $\mathcal{A}^{(i)}$ in epoch $i$. 
LEMMA 10. The difference between the expected cumulative loss of Algorithm 1 during epoch $i$, and the expected cumulative loss of $\mathcal{A}^{(i)}$ during that epoch, is bounded as

$$
\mathbb{E}\left[\sum_{t \in I_{i}} f_{t}\left(x_{t}\right)-\sum_{t \in I_{i}} f_{t}\left(z_{t}^{(i)}\right)\right] \leqslant \frac{4 \log (2 L T)}{\nu}+\nu T_{i} .
$$

Proof. The algorithm $\mathcal{A}$ is following $\mathrm{MW}^{1}$ updates over $m=\left\lceil\log _{2} L\right\rceil \cdot\left\lceil\log _{2} T\right\rceil$ algorithms as meta-experts. Thus, Lemma 11 gives

$$
\mathbb{E}\left[\sum_{t \in I_{i}} f_{t}\left(x_{t}\right)-\sum_{t \in I_{i}} f_{t}\left(z_{t}^{(i)}\right)\right] \leqslant \frac{2 \log (m T)}{\nu}+\nu T .
$$

Using $m \leqslant 2 L T$ to bound the logarithmic term gives the result.

We now turn to prove the theorem.

Proof of Theorem 6. First, regarding the running time of the algorithm, note that on each round Algorithm 1 has to update $O(\log (L) \log (T))$ instances of $\mathrm{MW}^{1}$, where each such update costs at most $O(\log L)$ time according to Lemma 12. Hence, the overall runtime per round is $O\left(\log ^{2}(L) \log (T)\right)$.

We next analyze the expected regret of the algorithm. Summing the bounds of Lemmas 9 and 10 over epochs $i=$ $1, \ldots, m$ and adding that of Lemma 7 , we can bound the expected regret of Algorithm 1 as follows:

$$
\begin{aligned}
& \mathbb{E}\left[\sum_{t=t_{0}+1}^{t_{1}} f_{t}\left(x_{t}\right)\right]-\left(\sum_{t=1}^{t_{1}} f_{t}\left(x_{t_{1}}^{\star}\right)-\sum_{t=1}^{t_{0}} f_{t}\left(x_{t_{0}}^{\star}\right)\right) \\
& \leqslant 10 \sqrt{\log (2 L T)} \sum_{i=1}^{m} \sqrt{k_{i} T_{i}}+\sum_{i=1}^{m}\left(\frac{4 \log (2 L T)}{\nu}+\nu T_{i}\right) \\
& \leqslant 10 \sqrt{\log (2 L T)} \sum_{i=1}^{m} \sqrt{k_{i} T_{i}}+\frac{4 L \log (2 L T)}{\nu}+\nu T
\end{aligned}
$$

where we have used $m \leqslant L$ and $\sum_{i=1}^{m} T_{i}=T$. In order to bound the sum on the right-hand side, we first notice that $\sum_{i=1}^{m} k_{i} \leqslant 3 \sum_{i=1}^{m}\left|C_{i}\right| \leqslant 3 L$. Hence, using the CauchySchwarz inequality we get $\sum_{i} \sqrt{k_{i} T_{i}} \leqslant \sqrt{\sum_{i} k_{i}} \sqrt{\sum_{i} T_{i}} \leqslant$ $\sqrt{3 L T}$. Combining this with Eq. (3) and our choice of $\nu$, and rearranging the left-hand side of the inequality, we obtain

$$
\begin{aligned}
& \mathbb{E}\left[\sum_{t=t_{0}+1}^{t_{1}} f_{t}\left(x_{t}\right)\right]-\left(\sum_{t=1}^{t_{1}} f_{t}\left(x_{t_{1}}^{\star}\right)-\sum_{t=1}^{t_{0}} f_{t}\left(x_{t_{0}}^{\star}\right)\right) \\
& \leqslant 25 \sqrt{L T \log (2 L T)},
\end{aligned}
$$

and the theorem follows.

\subsection{Main Algorithm}

We now ready to present our main online algorithm: an algorithm for online learning with optimizable experts, that guarantees $\epsilon$ expected average regret in total $\widetilde{O}\left(\sqrt{N} / \epsilon^{2}\right)$ time. The algorithm is presented in Algorithm 2, and in the following theorem we give its guarantees.

ThEOREM 1 (restated). The expected average regret of Algorithm 2 on any sequence of $T$ loss functions $f_{1}, \ldots, f_{T}$ : $[N] \mapsto[0,1]$ over $N$ experts is upper bounded by $40 N^{1 / 4} \log (N T) / \sqrt{T}$. The algorithm can be implemented to run in $\widetilde{O}(1)$ time per round in the optimizable experts model.
PARAmeters: $N, T$

1. Set $\eta=2 /\left(N^{1 / 4} \sqrt{T}\right)$ and $\nu=2 \sqrt{\log (2 T) / T}$

2. Sample a set $R$ of $\lfloor 2 \sqrt{N} \log T\rfloor$ experts uniformly at random with replacement

3. Initialize $\mathcal{A}_{1}=\mathrm{MW}^{2}\left(\eta, \frac{1}{T}\right)$ on the experts in $R$

4. Initialize $\mathcal{A}_{2}=\operatorname{LeAders}(L, T)$ with $L=\lfloor\sqrt{N}\rfloor$

5. Initialize $\mathcal{A}=\mathrm{MW}^{1}\left(\nu, \frac{1}{T}\right)$ on $\mathcal{A}_{1}$ and $\mathcal{A}_{2}$ as experts

6. For $t=1,2, \ldots, T$ :

(a) Play the prediction $x_{t}$ of $\mathcal{A}$

(b) Observe $f_{t}$ and the new leader $x_{t}^{\star}$, and use them to update $\mathcal{A}_{1}, \mathcal{A}_{2}$ and $\mathcal{A}$

\section{Algorithm 2: Algorithm for online learning with an optimization oracle.}

Algorithm 2 relies on the LEADERs and $\mathrm{MW}^{1}$ algorithms discussed earlier, and on yet another variant of the MW method - the $\mathrm{MW}^{2}$ algorithm - which is similar to $\mathrm{MW}^{1}$. The difference between the two algorithms is in their running time per round: $\mathrm{MW}^{1}$, like standard $\mathrm{MW}$, runs in $O(N)$ time per round over $N$ experts; $\mathrm{MW}^{2}$ is an "amortized" version of $\mathrm{MW}^{1}$ that spreads computation over time and runs in only $\widetilde{O}(1)$ time per round, but requires $N$ times more rounds to converge to the same average regret.

Lemma 12. Suppose that $\mathrm{MW}^{2}$ (see Algorithm 4) is used for prediction with expert advice, against an arbitrary sequence of loss functions $f_{1}, f_{2}, \ldots:[N] \mapsto[0,1]$ over $N$ experts. Then, for $\gamma=\frac{1}{T}$ and any $\eta>0$, its sequence of predictions $x_{1}, x_{2}, \ldots$ satisfies

$$
\mathbb{E}\left[\sum_{t=t_{0}}^{t_{1}} f_{t}\left(x_{t}\right)\right]-\min _{x \in[N]} \sum_{t=t_{0}}^{t_{1}} f_{t}(x) \leqslant \frac{4 \log (N T)}{\eta}+\eta N T
$$

in any time interval $\left\{t_{0}, \ldots, t_{1}\right\}$ of length at most $T$. The algorithm can be implemented to run in $O(\log N)$ time per round.

Given the LEADERS algorithm, the overall idea behind Algorithm 2 is quite simple: first guess $\sqrt{N}$ experts uniformly at random, so that with nice probability one of the "top" $\sqrt{N}$ experts is picked, where experts are ranked according to the last round of the game in which they are leaders. (In particular, the best expert in hindsight is ranked first.) The first online algorithm $\mathcal{A}_{1}$ - an instance of $\mathrm{MW}^{2}$ - is designed to compete with this leader, up to that point in time where it appears as leader for the last time. At this point, the second algorithm $\mathcal{A}_{2}$ - an instance of LEADERS - comes into action and controls the regret until the end of the game. It is able to do so because in that time period there are only few different leaders (i.e., at most $\sqrt{N}$ ), and as we pointed out earlier, LEADERS is designed to exploit this fact. The role of the algorithm $\mathcal{A}$, being executed on top of $\mathcal{A}_{1}$ and $\mathcal{A}_{2}$ as experts, is to combine between the two regret guarantees, each in its relevant time interval.

Using Theorem 6 and Lemmas 11 and 12, we can formalize the intuitive idea sketched above and prove the main result of this section.

Proof of Theorem 1 . The fact that the algorithm can be implemented to run in $\widetilde{O}(1)$ time per round follows immediately from the running time of the algorithms $\mathrm{MW}^{1}$, 
$\mathrm{MW}^{2}$, and LEADERs, each of which runs in $\widetilde{O}(1)$ time per round with the parameters used in Algorithm 2.

We move on to analyze the expected regret. Rank each expert $x \in[N]$ according to $\operatorname{rank}(x)=0$ if $x$ is never a leader throughout the game, and $\operatorname{rank}(x)=\max \left\{t: x_{t}^{\star}=x\right\}$ otherwise. Let $x_{(1)}, \ldots, x_{(N)}$ be the list of experts sorted according to their rank in decreasing order (with ties broken arbitrarily). In words, $x_{(1)}$ is the best expert in hindsight, $x_{(2)}$ is the expert leading right before $x_{(1)}$ becomes the sole leader, $x_{(3)}$ is the leading expert right before $x_{(1)}$ and $x_{(2)}$ become the only leaders, and so on. Using this definition, we define $X^{\star}=\left\{x_{(1)}, \ldots, x_{(n)}\right\}$ be the set of the top $n=\lfloor\sqrt{N}\rfloor$ experts having the highest rank.

First, consider the random set $R$. We claim that with high probability, this set contains at least one of the top $n$ leaders. Indeed, we have

$$
\begin{aligned}
\mathbb{P}\left(R \cap X^{\star}=\varnothing\right) & =\left(1-\frac{n}{N}\right)^{\lfloor 2 \sqrt{N} \log T\rfloor} \\
& \leqslant\left(1-\frac{1}{2 \sqrt{N}}\right)^{\sqrt{N} \log T} \\
& \leqslant e^{-\frac{1}{2} \log T}=\frac{1}{\sqrt{T}}
\end{aligned}
$$

so that with probability at least $1-1 / \sqrt{T}$ it holds that $R \cap X^{\star} \neq \varnothing$. As a result, it is enough to upper bound the expected regret of the algorithm for any fixed realization of $R$ such that $R \cap X^{\star} \neq \varnothing$ : in the event that the intersection is empty, that occurs with probability $1 / \sqrt{T}$, the regret can be at most $T$ and thus ignoring these realizations can only affect the expected regret by an additive $\sqrt{T}$ term. Hence, in what follows we fix an arbitrary realization of the set $R$ such that $R \cap X^{\star} \neq \varnothing$ and bound the expected regret of the algorithm.

Given $R$ with $R \cap X^{\star} \neq \varnothing$, we can pick $x \in R \cap X^{\star}$ and let $T_{0}=\operatorname{rank}(x)$ be the last round in which $x$ is the leader. Since $x \in R$ and $|R| \leqslant 2 \sqrt{N} \log T$, the $\mathrm{MW}^{2}$ instance $\mathcal{A}_{1}$ over the experts in $R$, with parameter $\eta=2 /\left(N^{1 / 4} \sqrt{T}\right)$, guarantees (recall Lemma 12) that

$$
\begin{aligned}
\mathbb{E}\left[\sum_{t=1}^{T_{0}} f_{t}\left(x_{t}^{(1)}\right)\right]-\sum_{t=1}^{T_{0}} f_{t}\left(x_{T_{0}}^{\star}\right) & \leqslant \frac{4 \log (|R| T)}{\eta}+\eta|R| T \\
& \leqslant 8 N^{1 / 4} \sqrt{T} \log (2 N T)
\end{aligned}
$$

where we use $x_{t}^{(1)}$ to denote the decision of $\mathcal{A}_{1}$ on round $t$.

On the other hand, observe that there are at most $n$ different leaders throughout the time interval $\left\{T_{0}+1, \ldots, T\right\}$, which follows from the fact that $x \in X^{\star}$. Thus, in light of Theorem 6, we have

$$
\begin{aligned}
& \mathbb{E}\left[\sum_{t=T_{0}+1}^{T} f_{t}\left(x_{t}^{(2)}\right)\right]-\left(\sum_{t=1}^{T} f_{t}\left(x_{T}^{\star}\right)-\sum_{t=1}^{T_{0}} f_{t}\left(x_{T_{0}}^{\star}\right)\right) \\
& \leqslant 25 N^{1 / 4} \sqrt{T \log (2 N T)},
\end{aligned}
$$

where here $x_{t}^{(2)}$ denotes the decision of $\mathcal{A}_{2}$ on round $t$.

Now, since Algorithm 2 is playing $\mathrm{MW}^{1}$ on $\mathcal{A}_{1}$ and $\mathcal{A}_{2}$ as experts with parameter $\nu=2 \sqrt{\log (2 T) / T}$, Lemma 11 shows that

$$
\begin{aligned}
\mathbb{E}\left[\sum_{t=1}^{T_{0}} f_{t}\left(x_{t}\right)-\sum_{t=1}^{T_{0}} f_{t}\left(x_{t}^{(1)}\right)\right] & \leqslant \frac{2 \log (2 T)}{\nu}+\nu T \\
& =3 \sqrt{T \log (2 T)}
\end{aligned}
$$

PARAmETERS: $\eta, \gamma$

1. Initialize $w_{1}(x)=1$ for all $x \in[N]$

2. For $t=1,2, \ldots$ :

(a) For all $x \in[N]$ compute $q_{t}(x)=w_{t}(x) / W_{t}$ with $W_{t}=\sum_{y} w_{t}(y)$

(b) Pick $x_{t} \sim q_{t}$, play $x_{t}$ and receive feedback $f_{t}$

(c) For all $x \in[N]$, update

$$
w_{t+1}(x)=w_{t}(x) e^{-\eta f_{t}(x)}+\frac{\gamma}{N} W_{t}
$$

\section{Algorithm 3: The $\mathrm{MW}^{1}$ algorithm.}

and similarly,

$$
\mathbb{E}\left[\sum_{t=T_{0}+1}^{T} f_{t}\left(x_{t}\right)-\sum_{t=T_{0}+1}^{T} f_{t}\left(x_{t}^{(2)}\right)\right] \leqslant 3 \sqrt{T \log (2 T)}
$$

Summing up Eqs. (4) to (7) we obtain the regret bound

$$
\mathbb{E}\left[\sum_{t=1}^{T} f_{t}\left(x_{t}\right)\right]-\sum_{t=1}^{T} f_{t}\left(x_{T}^{\star}\right) \leqslant 39 N^{1 / 4} \sqrt{T} \log (N T)
$$

for any fixed realization of $R$ with $R \cap X^{\star} \neq \varnothing$. As we explained before, the overall expected regret is larger by at most $\sqrt{T}$ than the right-hand side of Eq. (8), and dividing through by $T$ gives the theorem.

\subsection{Multiplicative Weights Algorithms}

We end the section by presenting the several variants of the Multiplicative Weights (MW) method used in our algorithms above. For an extensive survey of the basic MW method and its applications, refer to [6].

\subsection{1 $\mathrm{MW}^{1}$ : Mixed $M W$}

The first variant, the $\mathrm{MW}^{1}$ algorithm, is designed so that its regret on any time interval of bounded length is controlled. The standard MW algorithm does not have such a property, because the weight it assigns to an expert might become very small if this expert performs badly, so that even if the expert starts making good decisions, it cannot regain a non-negligible weight.

Our modification of the algorithm (see Algorithm 3) involves mixing in a fixed weight to the update of the algorithm, for all experts on each round, so as to keep the weights away from zero at all times. We note that this is not equivalent to the more standard modification of mixing-in the uniform distribution to the sampling distributions of the algorithm: in our variant, it is essential that the mixed weights are fed back into the update of the algorithm so as to control its weights.

In the following lemma we prove a regret bound for the $\mathrm{MW}^{1}$ algorithm. We prove a slightly more general result than the one we stated earlier in the section, which will become useful for the analysis of the subsequent algorithms. 
PARAMETERS: $\eta, \gamma$

1. Initialize $w_{1}(x)=1$ for all $x \in[N]$

2. For $t=1,2, \ldots$ :

(a) For all $x \in[N]$ compute $q_{t}(x)=w_{t}(x) / W_{t}$ with $W_{t}=\sum_{y} w_{t}(y)$

(b) Pick $x_{t} \sim q_{t}$, play $x_{t}$ and receive feedback $f_{t}$

(c) Pick $y_{t} \in[N]$ uniformly at random, and for all $x \in[N]$ update:

$$
w_{t+1}(x)= \begin{cases}w_{t}(x) e^{-\eta N f_{t}(x)}+\frac{\gamma}{N} W_{t} & \text { if } x=y_{t} \\ w_{t}(x)+\frac{\gamma}{N} W_{t} & \text { otherwise }\end{cases}
$$

\section{Algorithm 4: The $\mathrm{MW}^{2}$ algorithm.}

LEMma 11. For any sequence of loss functions $f_{1}, f_{2}, \ldots$ : $[N] \mapsto \mathbb{R}^{+}$and for $\gamma=\frac{1}{T}$ and any $\eta>0$, Algorithm 3 guarantees

$$
\begin{aligned}
\mathbb{E}\left[\sum_{t=t_{0}}^{t_{1}} f_{t}\left(x_{t}\right)\right] & -\min _{x \in[N]} \sum_{t=t_{0}}^{t_{1}} f_{t}(x) \\
& \leqslant \frac{2 \log (N T)}{\eta}+\eta \mathbb{E}\left[\sum_{t=t_{0}}^{t_{1}} f_{t}\left(x_{t}\right)^{2}\right]
\end{aligned}
$$

in any time interval $\left\{t_{0}, \ldots, t_{1}\right\}$ of length at most $T$. The algorithm can be implemented to run in $O(N)$ time per round.

The proof of the lemma, as well as the proofs of all subsequent lemmas in this section, are deferred to [29].

\subsection{2 $\mathrm{MW}^{2}$ : Amortized Mixed MW}

We now give an amortized version of the $\mathrm{MW}^{1}$ algorithm. Specifically, we give a variant of the latter algorithm that runs in $\widetilde{O}(1)$ per round and attains an $\widetilde{O}(\sqrt{N T})$ bound over the expected regret, as opposed to the $\mathrm{MW}^{1}$ algorithm that runs in time $O(N)$ per round and achieves $\widetilde{O}(\sqrt{T})$ regret. The algorithm, which we call $\mathrm{MW}^{2}$, is based on the $\mathrm{MW}^{1}$ update rule and incorporates sampling for accelerating the updates. ${ }^{6}$

For Algorithm 4 we prove:

LEMma 12. For any sequence of loss functions $f_{1}, f_{2}, \ldots$ : $[N] \mapsto[0,1]$, and for $\gamma=\frac{1}{T}$ and any $\eta>0$, the Algorithm 4 guarantees that

$$
\mathbb{E}\left[\sum_{t=t_{0}}^{t_{1}} f_{t}\left(x_{t}\right)\right]-\min _{x \in[N]} \sum_{t=t_{0}}^{t_{1}} f_{t}(x) \leqslant \frac{4 \log (N T)}{\eta}+\eta N T
$$

in any time interval $\left\{t_{0}, \ldots, t_{1}\right\}$ of length at most $T$. Furthermore, the algorithm can be implemented to run in $O(\log N)$ time per round.

\subsection{3 $\mathrm{MW}^{3}$ : Sliding Amortized Mixed MW}

The final component we require is a version of $\mathrm{MW}^{2}$ that works in an online learning setting with activated experts. In this version, on each round of the game one of the experts is "activated". The sequence of activations is determined based only on the loss values and does not depend on past

\footnotetext{
${ }^{6}$ This technique is reminiscent of bandit algorithms; however, notice that here we separate exploration and exploitation: we sample two experts on each round, instead of one as required in the bandit setting.
}

PARAMETERS: $k, \eta, \gamma$

1. Initialize $B_{1}(i)=i$ and $w_{1}(i)=1$ for all $i \in[k]$

2. For $t=1,2, \ldots$ :

(a) For all $i \in[k]$ compute $q_{t}(i)=w_{t}(i) / W_{t}$ with $W_{t}=$ $\sum_{y} w_{t}(i)$

(b) Pick $i_{t} \sim q_{t}$, play $x_{t}=B_{t}\left(i_{t}\right)$, receive $f_{t}$ and new activated expert $x_{t}^{\star}$

(c) Update weights: pick $j_{t} \in[k]$ uniformly at random, and for all $i \in[k]$ set:

$$
\begin{aligned}
& w_{t+1}(i)= \\
& \begin{cases}w_{t}(i) e^{-\eta N f_{t}\left(B_{t}(i)\right)}+\frac{\gamma}{N} W_{t} & \text { if } i=j_{t} \\
w_{t}(i)+\frac{\gamma}{N} W_{t} & \text { otherwise }\end{cases}
\end{aligned}
$$

(d) Update buffer: set $B_{t+1}=B_{t}$; if $x_{t}^{\star} \notin B_{t}$, find the index $i^{\prime} \in[k]$ of the oldest activated expert in $B_{t}$ (break ties arbitrarily) and set $B_{t+1}\left(i^{\prime}\right)=x_{t}^{\star}$.

\section{Algorithm 5: The $\mathrm{MW}^{3}$ algorithm.}

decisions of the algorithm; thus, it can be thought of as set by the oblivious adversary before the game starts. The goal of the player is to compete only with the last $k$ (distinct) activated experts, for some parameter $k$. In the context of the present section, the expert activated on round $t$ is the leader at the end of that round. Therefore, we overload notation and denote by $x_{t}^{\star}$ the expert activated on round $t$.

The $\mathrm{MW}^{3}$ algorithm, presented in Algorithm 5, is a "sliding window" version of $\mathrm{MW}^{2}$ that keeps a buffer of the last $k$ (distinct) activated experts. When its buffer gets full and a new expert is activated, the algorithm evicts from the buffer the expert whose most recent activation is the oldest. (Notice that the latter expert is not necessarily the oldest one in the buffer, as an expert can be re-activated while already in the buffer.) In this case, the newly inserted expert is assigned the same weight of the expert evicted from the buffer.

For the $\mathrm{MW}^{3}$ algorithm we prove:

LEMMA 13. Assume that expert $x^{\star} \in[N]$ was activated on round $t_{0}$, and from that point until round $t_{1}$ there were no more than $k$ different activated experts (including $x^{\star}$ itself). Then, for any time interval $\left[t_{0}^{\prime}, t_{1}^{\prime}\right] \subseteq\left[t_{0}, t_{1}\right]$ of length at most $T$, the $\mathrm{MW}^{3}$ algorithm with parameters $k, \gamma=\frac{1}{T}$ and any $\eta>0$ guarantees

$$
\mathbb{E}\left[\sum_{t=t_{0}^{\prime}+1}^{t_{1}^{\prime}} f_{t}\left(x_{t}\right)\right]-\sum_{t=t_{0}^{\prime}+1}^{t_{1}^{\prime}} f_{t}\left(x^{\star}\right) \leqslant \frac{4 \log (k T)}{\eta}+\eta k T .
$$

Furthermore, the algorithm can be implemented to run in time $\widetilde{O}(1)$ per round.

\section{SOLVING ZERO-SUM GAMES WITH BEST-RESPONSE ORACLES}

In this section we apply our online algorithms to repeated game playing in zero-sum games with best-response oracles. Before we do that, we first have to extend our results to the case where the assignment of losses can be adaptive to the decisions of the algorithm. Namely, unlike in the standard model of an oblivious adversary where the loss functions are being determined before the game begins, in the adaptive setting the loss function $f_{t}$ on round $t$ may depend on the 
(possibly randomized) decisions $x_{1}, \ldots, x_{t-1}$ chosen in previous rounds.

Fortunately, with minor modifications Algorithm 2 can be adapted to the non-oblivious setting and obtain low regret against adaptive adversaries as well. In a nutshell, we show that the algorithm can be made "self-oblivious", in the sense that its decision on each round depends only indirectly on its previous decisions, through its dependence on the previous loss functions; algorithms with this property are ensured to work well against adaptive adversaries [see 37, 14, 12]. Furthermore, the same property is also sufficient for the adapted algorithm to obtain low regret with high probability, and not only in expectation. The formal details are given in the proof of the following corollary.

COROLlary 14. With probability at least $1-\delta$, the average regret of Algorithm 2 (when implemented appropriately) is upper bounded by $40 N^{1 / 4} \log \left(\frac{N T}{\delta}\right) / \sqrt{T}$. This is true even when the algorithm faces a non-oblivious adversary.

Proof. We first explain how Algorithm 2 can be implemented so as the following "self-obliviousness" property holds, for all rounds $t$ :

$$
\begin{array}{r}
\mathbb{P}\left(x_{t}=x \mid x_{1}, \ldots, x_{t-1}, f_{1}, \ldots, f_{t-1}\right) \\
=\mathbb{P}\left(x_{t}=x \mid f_{1}, \ldots, f_{t-1}\right) .
\end{array}
$$

We can ensure this holds by randomizing separately for making the decisions $x_{t}$, and for updating the algorithm. That is, if $x_{t}$ is sampled from a distribution $p_{t}$ on round $t$ of the game, then we discard $x_{t}$ and use a different independent sample $x_{t}^{\prime} \sim p_{t}$ for updating the algorithm. In fact, Algorithm 2 makes multiple updates on each round (to the various $\mathcal{A}_{r, s}$ algorithms, etc.); we ensure that the sample $x_{t}$ is not used in any of these updates, and a fresh sample is picked when necessary. Further, we note that this slight modification does not impact the runtime of the algorithm, up to constants.

With Algorithm 2 implemented this way, we can now use, e.g., Lemma 4.1 of Cesa-Bianchi and Lugosi [12] to obtain from Theorem 1 that

$$
\begin{aligned}
\frac{1}{T} \sum_{t=1}^{T} f_{t}\left(x_{t}\right) & -\min _{x \in[N]} \frac{1}{T} \sum_{t=1}^{T} f_{t}(x) \\
& \leqslant \frac{40 N^{1 / 4} \log (N T)}{\sqrt{T}}+\sqrt{\frac{\log \frac{1}{\delta}}{2 T}}
\end{aligned}
$$

holds with probability at least $1-\delta$ against any non-oblivious adversary. Further upper bounding the right-hand side of the above yields the stated regret bound.

Using standard techniques [21], we can now use Algorithm 2 to solve zero-sum games equipped with bestresponse oracles. The simple scheme is presented in Algorithm 6: both players use the online Algorithm 2 to produce their decisions throughout the game, and employ their best response oracles to compute the "leaders". In the context of zero-sum games, the leader on iteration $t$ is the best response to the empirical distribution of the past plays of the opponent.

We remark that, in fact, it is sufficient that only one of the players follow Algorithm 2 for ensuring fast convergence to equilibrium. The other player could, for example, behave greedily and simply follow his best response to the plays of the first player - a strategy known as "fictitious play".
PARAMETERS: game matrix $G \in[0,1]^{N \times N}$, parameter $T$

1. Initialize instances $\mathcal{A}_{1}, \mathcal{A}_{2}$ of Algorithm 2 with parameters $N, T$ for the row and column players, respectively

2. For $t=1,2, \ldots, T$ :

(a) Let the players play the decisions $x_{t}, y_{t}$ of $\mathcal{A}_{1}, \mathcal{A}_{2}$, respectively

(b) Let $\bar{p}_{t}$ be the empirical distribution of $x_{1}, \ldots, x_{t}$, and let $\bar{q}_{t}$ be the empirical distribution of $y_{1}, \ldots, y_{t}$

(c) Update $\mathcal{A}_{1}$ with the loss function $G\left(\cdot, y_{t}\right)$ and the leader $x_{t}^{\star}=\mathrm{BR}^{1}\left(\bar{q}_{t}\right)$

(d) Update $\mathcal{A}_{2}$ with the loss function $G\left(x_{t}, \cdot\right)$ and the leader $y_{t}^{\star}=\operatorname{BR}^{2}\left(\bar{p}_{t}\right)$

OutPut: the profile $(\bar{p}, \bar{q})=\left(\bar{p}_{T}, \bar{q}_{T}\right)$

\section{Algorithm 6: Algorithm for zero-sum games with} best-response oracles.

\section{For Algorithm 6 we prove the following.}

Theorem 3 (restated). With probability at least $1-\delta$, Algorithm 6 with $T=\frac{240^{2} \sqrt{N}}{\epsilon^{2}} \log ^{2} \frac{240 N}{\epsilon \delta}$ outputs a mixed strategy profile $(\bar{p}, \bar{q})$ being an $\epsilon$-approximate equilibrium. The algorithm can be implemented to run in $\widetilde{O}\left(\sqrt{N} / \epsilon^{2}\right)$ time.

Proof. First, we note the running time. Notice that the empirical distributions $\bar{p}_{t}$ and $\bar{q}_{t}$ do not have to be recomputed each round, and can be maintained incrementally with constant time per iteration. Furthermore, since we assume that each call to the best response oracles costs $O(1)$ time, Theorem 1 shows that the updates of $\mathcal{A}_{1}, \mathcal{A}_{2}$ can be implemented in time $\widetilde{O}(1)$. Hence, each iteration costs $\widetilde{O}(1)$ and so the overall runtime is $\widetilde{O}\left(\sqrt{N} / \epsilon^{2}\right)$.

We move on to analyze the output of the algorithm. Using the regret guarantee of Corollary 14 for the online algorithm of the row player, we have

$\frac{1}{T} \sum_{t=1}^{T} G\left(x_{t}, y_{t}\right)-\min _{x \in[N]} \frac{1}{T} \sum_{t=1}^{T} G\left(x, y_{t}\right) \leqslant \frac{40 N^{1 / 4}}{\sqrt{T}} \log \frac{2 N T}{\delta}$

with probability at least $1-\frac{\delta}{2}$. Similarly, for the online algorithm of the column player we have

$\max _{y \in[N]} \frac{1}{T} \sum_{t=1}^{T} G\left(x_{t}, y\right)-\frac{1}{T} \sum_{t=1}^{T} G\left(x_{t}, y_{t}\right) \leqslant \frac{40 N^{1 / 4}}{\sqrt{T}} \log \frac{2 N T}{\delta}$

with probability at least $1-\frac{\delta}{2}$. Hence, summing the two inequalities and using $\frac{1}{T} \sum_{t=1}^{T^{2}} G\left(x_{t}, y\right)=G(\bar{p}, y)$ and $\frac{1}{T} \sum_{t=1}^{T} G\left(x, y_{t}\right)=G(x, \bar{q})$, we have

$$
\max _{q \in \Delta_{N}} G(\bar{p}, q)-\min _{p \in \Delta_{N}} G(p, \bar{q}) \leqslant \frac{80 N^{1 / 4}}{\sqrt{T}} \log \frac{N T}{\delta} \leqslant \epsilon
$$

with probability at least $1-\delta$; the ultimate inequality involves our choice of $T$ and a tedious calculation.

Now, let $\left(p^{\star}, q^{\star}\right)$ be an equilibrium of the game, and denote by $\lambda^{\star}=G\left(p^{\star}, q^{\star}\right)$ the value of the game. As a result of Eq. (10), for all $q \in \Delta_{N}$ we have

$$
\begin{aligned}
G(\bar{p}, q) & \leqslant \min _{p \in \Delta_{N}} G(p, \bar{q})+\epsilon \\
& \leqslant G\left(p^{\star}, \bar{q}\right)+\epsilon \\
& \leqslant G\left(p^{\star}, q^{\star}\right)+\epsilon=\lambda^{\star}+\epsilon .
\end{aligned}
$$


Similarly, for all $p \in \Delta_{N}$,

$$
\begin{aligned}
G(p, \bar{q}) & \geqslant \max _{q \in \Delta_{N}} G(\bar{p}, q)-\epsilon \\
& \geqslant G\left(\bar{p}, q^{\star}\right)-\epsilon \\
& \geqslant G\left(p^{\star}, q^{\star}\right)-\epsilon=\lambda^{\star}-\epsilon .
\end{aligned}
$$

This means that, with probability at least $1-\delta$, the mixed strategy profile $(\bar{p}, \bar{q})$ is an $\epsilon$-approximate equilibrium.

\section{LOWER BOUNDS}

In this section we prove our computational lower bounds for optimizable experts and learning in games, stated in Theorems 2 and 4 . We begin with the latter, and then prove Theorem 2 via reduction.

ThEOREM 4 (restated). For any efficient (randomized) players in a repeated $N \times N$ zero-sum game with bestresponse oracles, there exists a game matrix with $[0,1]$ values such that with probability at least $\frac{2}{3}$, the average payoff of the players is at least $\frac{1}{4}$ far from equilibrium after $\Omega\left(\sqrt{N} / \log ^{3} N\right)$ time.

We prove Theorem 4 by means of a reduction from a local-search problem studied by Aldous [5], which we now describe.

\section{Lower bounds for local search.}

Consider a function $f:\{0,1\}^{d} \mapsto \mathbb{N}$ over the $d$-dimensional hypercube. A local optimum of $f$ over the hypercube is a vertex such that the value of $f$ at this vertex is larger than or equal to the values of $f$ at all neighboring vertices (i.e., those with hamming distance one). A function is said to be globally-consistent if it has a single local optimum (or in other words, if every local optimum is also a global optimum of the function).

Aldous [5] considered the following problem (slightly rephrased here for convenience), which we refer to as Aldous' problem: Given a globally-consistent function $f:\{0,1\}^{d} \mapsto$ $\mathbb{N}$ (given as a black-box oracle), determine whether the maximal value of $f$ is even or odd with a minimal number of queries to the function.

The following theorem is an improvement of Aaronson [1] to a result initially proved by Aldous [5].

Theorem 15 (Aldous [5], Aaronson [1]). For any randomized algorithm for Aldous' problem that makes no more than $\Omega\left(2^{d / 2} / d^{2}\right)$ value queries in the worst case, there exists a function $f:\{0,1\}^{d} \mapsto \mathbb{N}$ such that the algorithm cannot determine with probability higher than $\frac{2}{3}$ whether the maximal value of $f$ over $\{0,1\}^{d}$ is even or odd.

We reduce Aldous' problem to the problem of approximately solving a large zero-sum game with best-response and value oracles. Our reduction proceeds by constructing a specific form of a game, which we now describe.

\section{The reduction.}

Let $f:[N] \mapsto \mathbb{N}$ be an input to Aldous' problem, with maximal value $f^{\star}=\max _{i \in[N]} f(i)$. (Here, we identify each vertex of the $\left\lceil\log _{2} N\right\rceil$-dimensional hypercube with a natural number in the range $1, \ldots, N$ corresponding to its binary representation.) We shall describe a zero-sum game with value $\lambda=\lambda\left(f^{\star}\right)$, where

$$
\forall k \in \mathbb{N}, \quad \lambda(k)= \begin{cases}\frac{1}{4} & \text { if } k \text { is even, } \\ \frac{3}{4} & \text { if } k \text { is odd. }\end{cases}
$$

Henceforth, we use $\Gamma(V)$ to denote the set of neighbors of a set of vertices $V \subseteq[N]$ of the hypercube (that includes the vertices in $V$ themselves).

The game matrix and its corresponding oracles are constructed as follows:

- Game matrix: Based on the function $f$, define a matrix $G^{f} \in[0,1]^{N \times N}$ as follows:

$$
G_{i, j}^{f}= \begin{cases}\lambda(f(i)) & \text { if } i \text { and } j \text { are local maxima of } f \\ 0 & \text { if } f(i) \geqslant f(j) \\ 1 & \text { otherwise. }\end{cases}
$$

- Value oracle: The oracle $\operatorname{Val}(i, j)$ simply returns $G_{i, j}^{f}$ for any $i, j \in[N]$ given as input.

- Best-response oracles: For any mixed strategy $p \in \Delta_{N}$, define:

$$
\mathrm{BR}^{1}(p)=\mathrm{BR}^{2}(p)=\underset{i \in \Gamma(\operatorname{supp}(p))}{\arg \max } f(i) .
$$

We set to prove Theorem 4. First, we assert that the value of the described game indeed equals $\lambda$.

LEMma 16. The minimax value of the game described by the matrix $G^{f}$ equals $\lambda$.

Proof. Let $i^{\star}=\arg \max _{i \in[N]} f(i)$ be the global maxima of $f$. We claim that the (pure) strategy profile $\left(i^{\star}, i^{\star}\right)$ is an equilibrium of the game given by $G^{f}$. To see this, notice that the payoff with this profile is $\lambda\left(f\left(i^{\star}\right)\right)=\lambda$. However, any profile of the form $\left(i, i^{\star}\right)$ generates a payoff of either $G_{i, i^{\star}}^{f}=\lambda$ or $G_{i, i^{\star}}^{f}=1$, since $f(i) \leqslant f\left(i^{\star}\right)$ for each $i$. Hence, the row player does not benefit by deviating from playing $i^{\star}$. Similarly, a profile of the form $\left(i^{\star}, j\right)$ yields a payoff of at most 0 , thus a deviation from $i^{\star}$ is not profitable to the column player either.

Next, we show that the value and best-response oracles we specified are correct.

LEMMA 17. The procedures $\mathrm{Val}$ and $\mathrm{BR}^{1}, \mathrm{BR}^{2}$ are correct value and best-response oracles for the game $G^{f}$.

Proof. The oracle Val is trivially a valid value oracle for the described game, as $\operatorname{Val}(i, j)=G_{i, j}^{f}$ for all $i, j \in[N]$ by definition. Moving on to the best-response oracles, we shall prove the claim for the oracle $\mathrm{BR}^{1}$; the proof for $\mathrm{BR}^{2}$ is very similar and thus omitted.

Consider some input $p \in \Delta_{N}$ and denote $j=\mathrm{BR}^{1}(p)$ the output of the oracle. Note that if $i^{\star} \in \operatorname{supp}(p)$, then $j=$ $\arg \max \{f(i): i \in \Gamma(\operatorname{supp}(p))\}=i^{\star}$ as $f$ has a single global maxima at $i^{\star}$. The main observation in this case is that the strategy $j=i^{\star}$ dominates all other column strategies $j^{\prime}$ : indeed, it is not hard to verify that since $f(j) \geqslant f\left(j^{\prime}\right)$ for any $j^{\prime}$, we have $G_{i, j}^{f} \geqslant G_{i, j^{\prime}}^{f}$ for all $j^{\prime}$. This implies that $p^{\top} G^{f} e_{j} \geqslant p^{\top} G^{f} e_{j^{\prime}}$ for all $j^{\prime}$, namely, $j$ is a best-response to $p$.

On the other hand, if $i^{\star} \notin \operatorname{supp}(p)$ we claim that $p^{\top} G^{f} e_{j}=$ 1 , which would immediately give $p^{\top} G^{f} e_{j} \geqslant p^{\top} G^{f} e_{j^{\prime}}$ for all 
$j^{\prime}$ (as the maximal payoff in the game is 1). This follows because if $i^{\star} \notin \operatorname{supp}(p)$, then it must hold that $f(j)>f(i)$ for all $i \in \operatorname{supp}(p)$, which means that $G_{i, j}^{f}=1$ for all $i \in$ $\operatorname{supp}(p)$. Hence, $p^{\top} G^{f} e_{j}=1$ as claimed.

We can now prove our main theorem.

Proof of Theorem 4. Let $f:[N] \mapsto \mathbb{N}$ be an arbitrary globally-consistent function over the hypercube. Consider some algorithm that computes the value of the zero-sum game $G^{f}$ up to an additive error of $\frac{1}{4}$ with probability at least $\frac{2}{3}$. Determining the game value up to $\frac{1}{4}$ gives the value of $\lambda$ (that can equal either $\frac{1}{4}$ or $\frac{3}{4}$ ), which by construction is determined according to the maximal value of $f$ being even or odd. Thus, the number of queries to $f$ the algorithm makes, through one of the oracles, is lower-bounded by $\Omega\left(\sqrt{N} / \log ^{2} N\right)$ according to Theorem 15 . In what follows, we show how this lower bound can be translated to a lower bound on the runtime of the algorithm.

First, notice that the runtime of the algorithm is lower bounded by the total number of row/column indices touched by the algorithm, namely, the total number of indices that appear in inputs to one of the oracles Val, Opt at some point throughout the execution of the algorithm (we think of index $i$ as appearing in the input of the call $\operatorname{Opt}(p)$ if $i \in \operatorname{supp}(p))$. Hence, if we let $S$ denote the set of all indices touched by the algorithm, then it is enough to lower bound the size of $S$ in order to obtain a lower bound on the runtime of the algorithm.

Now, notice that the set of all entries of $f$ queried by the algorithm throughout its execution, via one of the oracles $\mathrm{Val}$ and Opt, is a subset of $\cup_{i \in S} \Gamma(i)$. Indeed, upon any index $i \in S$ that appears in the input to one of the oracles, the function $f$ has to be queried only at the neighborhood $\Gamma(i)$ to produce the required output (recall the definitions of the oracles in our construction of $G_{f}$ above). Hence, the total number of distinct queries to $f$ made by the algorithm is at most $O(|S| \cdot \log N)$. On the other hand, as we noted earlier, this number is lower bounded by $\Omega\left(\sqrt{N} / \log ^{2} N\right)$ as a result of Theorem 15. Both bounds together yield the lower bound $|S|=\Omega\left(\sqrt{N} / \log ^{3} N\right)$, which directly implies the desired runtime lower bound.

Now, we can obtain Theorem 2 as a direct corollary of the lower bound for zero-sum games. We remark that it is possible to prove a tighter lower bound than the one we prove here, via direct information-theoretic arguments; we defer details to [29].

Theorem 2 (restated). Any (randomized) algorithm in the optimizable experts model cannot guarantee an expected average regret smaller than $1 / 16$ over at least $T \geqslant 20$ rounds in total time better than $O\left(\sqrt{N} / \log ^{3} N\right)$.

Proof. Suppose that there exists an online algorithm in the optimizable experts model that guarantees expected average regret $<\frac{1}{16}$ in total time $\tau$, where $\tau=o\left(\sqrt{N} / \log ^{3} N\right)$. Following the line of arguments presented in Section 4, we can show that the algorithm can be used to approximate zero-sum games. First, as explained in Section 4, we may assume that the algorithm is self-oblivious (see that section for the definition), and for such an algorithm Lemma 4.1 in [12] shows that with probability at least $1-\delta=\frac{5}{6}$, the average regret after $T \geqslant 20$ rounds is at most $\frac{1}{16}+\sqrt{\log (1 / \delta) / 2 T} \leqslant \frac{1}{8}$. Then, following the proof of Theorem 3 we can show that the online algorithm, if deployed by two players in a zerosum game, can be used to approximate the equilibrium of the game to within $\frac{1}{4}$ with probability at least $\frac{2}{3}$ and, with access to best response oracles, in total runtime $O(\tau)$. This is a contradiction to the statement of Theorem 4, proving our claim.

\section{ACKNOWLEDGMENTS}

The authors would like to thank Robert Schapire for illuminating conversations and helpful remarks throughout this research, Uriel Feige for providing us with references on local search problems, and John Langford for crystalizing the connections to oracle-based contextual learning.

The research leading to these results has received funding from the European Union's Seventh Framework Programme (FP7/2007-2013 under grant agreement 336078ERC-SUBLRN), the National Science Foundation under Grant No. IIS-1523815, and a Google research award.

\section{References}

[1] S. Aaronson. Lower bounds for local search by quantum arguments. SIAM Journal on Computing, 35(4):804824,2006

[2] I. Adler. The equivalence of linear programs and zerosum games. International Journal of Game Theory, 42 (1):165-177, 2013.

[3] A. Agarwal. Computational Trade-offs in Statistical Learning. PhD thesis, EECS Department, University of California, Berkeley, 2012.

[4] A. Agarwal, D. Hsu, S. Kale, J. Langford, L. Li, and R. Schapire. Taming the monster: A fast and simple algorithm for contextual bandits. In Proceedings of the 31st International Conference on Machine Learning (ICML'14), pages 1638-1646, 2014.

[5] D. Aldous. Minimization algorithms and random walk on the $d$-cube. The Annals of Probability, pages 403$413,1983$.

[6] S. Arora, E. Hazan, and S. Kale. The multiplicative weights update method: a meta-algorithm and applications. Theory of Computing, 8(6):121-164, 2012.

[7] B. Awerbuch and R. Kleinberg. Online linear optimization and adaptive routing. J. Comput. Syst. Sci., 74(1): 97-114, 2008

[8] A. Blum. Separating distribution-free and mistakebound learning models over the boolean domain. In Proceedings of the 31st Annual Symposium on Foundations of Computer Science, pages 211-218. IEEE, 1990.

[9] A. Blum and Y. Mansour. From external to internal regret. Journal of Machine Learning Research, 8:13071324, 2007.

[10] F. Brandt, F. Fischer, and P. Harrenstein. On the rate of convergence of fictitious play. Theory of Computing Systems, 53(1):41-52, 2013.

[11] G. W. Brown. Iterative solution of games by fictitious play. Activity analysis of production and allocation, 13 (1):374-376, 1951. 
[12] N. Cesa-Bianchi and G. Lugosi. Prediction, Learning, and Games. Cambridge University Press, 2006.

[13] K. L. Clarkson, E. Hazan, and D. P. Woodruff. Sublinear optimization for machine learning. J. $A C M, 59(5)$ : 23:1-23:49, 2012.

[14] V. Dani and T. P. Hayes. Robbing the bandit: Less regret in online geometric optimization against an adaptive adversary. In Proceedings of the seventeenth annual ACM-SIAM symposium on Discrete algorithm, pages 937-943. Society for Industrial and Applied Mathematics, 2006.

[15] C. Daskalakis and Q. Pan. A counter-example to karlin's strong conjecture for fictitious play. In Foundations of Computer Science (FOCS), 2014 IEEE 55th Annual Symposium on, pages 11-20. IEEE, 2014.

[16] C. Daskalakis, A. Deckelbaum, and A. Kim. Nearoptimal no-regret algorithms for zero-sum games. In Proceedings of the Twenty-second Annual ACM-SIAM Symposium on Discrete Algorithms, SODA '11, pages 235-254, 2011.

[17] M. Dudík, D. Hsu, S. Kale, N. Karampatziakis, J. Langford, L. Reyzin, and T. Zhang. Efficient optimal learning for contextual bandits. In UAI 2011, Proceedings of the Twenty-Seventh Conference on Uncertainty in Artificial Intelligence, Barcelona, Spain, July 14-17, 2011, pages 169-178, 2011.

[18] E. Even-Dar, S. M. Kakade, and Y. Mansour. Online markov decision processes. Math. Oper. Res., 34(3): 726-736, 2009.

[19] E. Even-dar, Y. Mansour, and U. Nadav. On the convergence of regret minimization dynamics in concave games. In Proceedings of the Forty-first Annual ACM Symposium on Theory of Computing, STOC '09, pages 523-532, New York, NY, USA, 2009. ACM.

[20] Y. Freund and R. E. Schapire. A decision-theoretic generalization of on-line learning and an application to boosting. J. Comput. Syst. Sci., 55(1):119-139, 1997.

[21] Y. Freund and R. E. Schapire. Adaptive game playing using multiplicative weights. Games and Economic Behavior, 29(1):79-103, 1999.

[22] Y. Freund, R. E. Schapire, Y. Singer, and M. K. Warmuth. Using and combining predictors that specialize. In Proceedings of the twenty-ninth annual ACM symposium on Theory of computing, pages 334-343. ACM, 1997.

[23] E. Gofer, N. Cesa-Bianchi, C. Gentile, and Y. Mansour. Regret minimization for branching experts. In COLT'13, pages 618-638, 2013.

[24] M. D. Grigoriadis and L. G. Khachiyan. A sublineartime randomized approximation algorithm for matrix games. Operations Research Letters, 18:53-58, 1995.

[25] J. Hannan. Approximation to bayes risk in repeated play. In M. Dresher, A. W. Tucker, and P. Wolfe, editors, Contributions to the Theory of Games, volume 3, pages 97-139, 1957.

[26] S. Hart and A. Mas-Colell. A simple adaptive procedure leading to correlated equilibrium. Econometrica, 68(5): 1127-1150, 2000.
[27] E. Hazan. Introduction to Online Convex Optimization. 2014. URL http://ocobook.cs.princeton.edu/.

[28] E. Hazan and S. Kale. Online submodular minimization. J. Mach. Learn. Res., 13(1):2903-2922, 2012.

[29] E. Hazan and T. Koren. The computational power of optimization in online learning. arXiv preprint arXiv:1504.02089, 2015.

[30] E. Hazan, S. Kale, and S. Shalev-Shwartz. Near-optimal algorithms for online matrix prediction. In COLT, pages 38.1-38.13, 2012.

[31] D. P. Helmbold and M. K. Warmuth. Learning permutations with exponential weights. J. Mach. Learn. Res., 10:1705-1736, 2009.

[32] S. Kakade and A. T. Kalai. From batch to transductive online learning. In Advances in Neural Information Processing Systems, pages 611-618, 2006.

[33] S. M. Kakade, A. T. Kalai, and K. Ligett. Playing games with approximation algorithms. SIAM J. Comput., 39(3):1088-1106, 2009.

[34] A. Kalai and S. Vempala. Efficient algorithms for online decision problems. Journal of Computer and System Sciences, 71(3):291-307, 2005.

[35] R. Kleinberg, A. Niculescu-Mizil, and Y. Sharma. Regret bounds for sleeping experts and bandits. Machine learning, 80(2-3):245-272, 2010.

[36] N. Littlestone and M. K. Warmuth. The weighted majority algorithm. Information and Computation, 108 (2):212-261, 1994. ISSN 0890-5401.

[37] H. B. McMahan and A. Blum. Online geometric optimization in the bandit setting against an adaptive adversary. In Learning theory, pages 109-123. Springer, 2004 .

[38] Y. Nesterov. Smooth minimization of non-smooth functions. Mathematical programming, 103(1):127-152, 2005 .

[39] N. Nisan, T. Roughgarden, E. Tardos, and V. V. Vazirani. Algorithmic Game Theory. Cambridge University Press, New York, NY, USA, 2007. ISBN 0521872820.

[40] J. Robinson. An iterative method of solving a game. Annals of Mathematics, 54(2):296-301, 1951.

[41] S. Shalev-Shwartz and N. Srebro. SVM optimization: Inverse dependence on training set size. In Proceedings of the 25th International Conference on Machine Learning, ICML '08, pages 928-935, 2008.

[42] S. Shalev-Shwartz, O. Shamir, and E. Tromer. Using more data to speed-up training time. In AISTATS, volume 22, pages 1019-1027, 2012.

[43] M. Warmuth and D. Kuzmin. Online variance minimization. In G. Lugosi and H. Simon, editors, Learning Theory, volume 4005 of Lecture Notes in Computer Science, pages 514-528. Springer Berlin Heidelberg, 2006.

[44] M. Zinkevich. Online convex programming and generalized infinitesimal gradient ascent. In Proceedings of the 20th International Conference on Machine Learning (ICML-03), pages 928-936, 2003. 